\title{
Helical multiferroics for electric field controlled quantum information processing
}

\author{
M. Azimi,,${ }^{1,2}$ L. Chotorlishvili, ${ }^{1}$ S. K. Mishra, ${ }^{1}$ S. Greschner,${ }^{3}$ T. Vekua,${ }^{3}$ and J. Berakdar ${ }^{1}$ \\ ${ }^{1}$ Institut für Physik, Martin-Luther-Universität Halle-Wittenberg, 06120 Halle, Germany \\ ${ }^{2}$ Max-Planck Institute for Microstructure Physics, Weinberg 2, 06120 Halle, Germany \\ ${ }^{3}$ Institut für Theoretische Physik, Leibniz Universität Hannover, 30167 Hannover, Germany
}

(Received 21 November 2013; revised manuscript received 14 January 2014; published 30 January 2014)

\begin{abstract}
Magnetoelectric coupling in helical multiferroics allows us to steer spin order with electric fields. Here we show theoretically that in a helical multiferroic chain quantum information processing as well as quantum phases are highly sensitive to electric $(E)$ field. Applying $E$ field, the quantum state transfer fidelity can be increased and made directionally dependent. We also show that $E$ field transforms the spin-density-wave/nematic or multipolar phases of a frustrated ferromagnetic spin- $\frac{1}{2}$ chain in chiral phase with a strong magnetoelectric coupling. We find sharp reorganization of the entanglement spectrum as well as a large enhancement of fidelity susceptibility at Ising quantum phase transition from nematic to chiral states driven by electric field. These findings point to a tool for quantum information with low power consumption.
\end{abstract}

DOI: 10.1103/PhysRevB.89.024424

PACS number(s): 75.85.+t, 03.67.Bg, 75.10.Pq

\section{INTRODUCTION}

Multiferroics (MF) are materials that show simultaneously multiple spontaneous ferroic ordering [1]. Intrinsic coupling between the order parameters, e.g., ferromagnetism (FM), ferroelectricity $(\mathrm{FE})$, and/or ferroelasticity (for an overview we refer to Refs. [2-21]), allows for multifunctionality of devices with qualitatively new conceptions [10,22-25]. Particulary advantageous is the high sensitivity of some MF compounds to external fields [26-30]. This allows us to steer, for instance, magnetic order with moderate electric fields opening thus the door for magnetoelectric spintronics and spin-based information processing with ultra low power consumption and dissipation [10,23-25]. These prospects are fueled by advances in synthesis and nanofabrication which renders feasible versatile MF nano- and quantum structures with enhanced multiferroic coupling [2-6,31]. From a fundamental point of view MF are also fascinating as their properties often emerge from an interplay of competing exchange and electronic correlation, crystal symmetry, and coupled spin-charge dynamics. For example, the perovskite multiferroics $R \mathrm{MnO}_{3}$ with $R=\mathrm{Tb}, \mathrm{Dy}, \mathrm{Gd}$ and $\mathrm{Eu}_{1-x} \mathrm{Y}_{x}$ exhibit an incommensurate chiral spin order [32] coupled to a finite FE polarization. The underlying physics is governed by competing exchange and Dzyaloshiskii-Moriya (DM) [33] interactions: The spin-orbital coupling associated with $d(p)$ orbitals of magnetic (oxygen) ions triggers the FE polarization [34,35] $\mathbf{P} \propto \hat{e}_{i j} \times\left(\mathbf{S}_{i} \times \mathbf{S}_{j}\right)$; $\hat{e}_{i j}$ is a unit vector connecting the sites $i$ and $j$ at which the effective spins $\mathbf{S}_{i / j}$ reside (e.g., along the [110] direction for $\mathrm{TbMnO}_{3}$ ). $\mathbf{P}$ is thus linked with the spin order chirality $\kappa=\left(\mathbf{S}_{i} \times \mathbf{S}_{j}\right)$, offering thus a tool for electrical control of $\kappa$ because, as shown experimentally [36], $\mathbf{P}$ can be steered with an external electric field $E$ (with $|E| \sim 1 \mathrm{kV} / \mathrm{cm}$ ). Indeed, effects of magnetoelectric coupling (ME) are evident in the dynamical response to moderate $E$ [37-42], i.e., phenomena rooted in ME can be driven, and possibly controlled, by moderate external fields. It is noteworthy that the chiral behavior of $\mathrm{TbMnO}_{3}$ persists with miniaturization down to $6 \mathrm{~nm}$ [31]. Furthermore, the feasibility was demonstrated of a multiferroic spin- $\frac{1}{2}$ chain of $\mathrm{LiCu}_{2} \mathrm{O}_{2}$ [43] and field-switchable $\mathrm{LiCuVO}_{4}[44]$.
These facts combined with the robust topological nature of the intrinsic chirality are the key elements of the present proposal to utilize chiral MF for $E$-field controlled, spin-based quantum information processing. Starting from an established model [35] for chiral MF with the aim to inspect electrically driven quantum information processing and quantum phases in a multiferroic chain, we find that an applied electric field with strength $E \sim 10^{3} \mathrm{kV} / \mathrm{cm}$ increases strongly the quantum state transfer fidelity making it directionally dependent. The system can be steered electrically between spin-density-wave, nematic, multipolar, and chiral phases. We find that the $E$ field modifies drastically the entanglement spectrum and enhances the fidelity susceptibility at the Ising quantum phase transition from nematic to chiral states.

\section{THEORETICAL FRAMEWORK}

We employ an effective model with frustrated spin interaction for the description of a one-dimensional MF chain along the $x$ axis [35]. The chain is subjected to an electric ( $E$, applied along the $y$ axis) and a magnetic ( $B$ along the $z$ axis) field. The Hamiltonian reads

$$
\hat{H}=J_{1} \sum_{i=1} \mathbf{S}_{i} \mathbf{S}_{i+1}+J_{2} \sum_{i=1} \mathbf{S}_{i} \mathbf{S}_{i+2}-B \sum_{i=1} S_{i}^{z}-\mathbf{E} \widehat{\mathbf{P}} .
$$

The exchange interaction constant between nearest neighbor spins is chosen FM $J_{1}<0$, while next-nearest interaction is antiferromagnetic $J_{2}>0$. Below, we use units in which $J_{2}=$ 1 (typical values, e.g., for $\mathrm{LiCu}_{2} \mathrm{O}_{2}$ are $J_{1} \approx-11 \pm 3 \mathrm{meV}$; and $J_{2} \approx 7 \pm 1 \mathrm{meV}$ [45-48]). Equation (1) is an effective Hamiltonian based on the conditions that $E(B)$ fields are weak such that their direct coupling to electronic orbital motion is negligible. The classical $E$ field couples (with a strength $g_{M E}$ ) to the induced polarization, i.e.,

$$
\mathbf{E} \widehat{\mathbf{P}}=E g_{M E} \sum_{i}\left(\mathbf{S}_{i} \times \mathbf{S}_{i+1}\right)^{z} .
$$

While $\mathbf{S}_{i}$ will be treated fully quantum mechanically, displacements will not be quantized [42]. The quantity

$$
\kappa=\left\langle\kappa_{i}\right\rangle=\left\langle\left(\mathbf{S}_{i} \times \mathbf{S}_{i+1}\right)^{z}\right\rangle
$$


is known as the $z$ component of vector chirality (VC), which for brevity we call chirality.

The frustrated $J_{1}-J_{2}$ spin- $\frac{1}{2}$ chain was studied extensively both theoretically [49-55] (exhibiting its rich ground state phase diagram hosting multipolar and chiral phases) and experimentally [56-58]. However, neither the control of quantum information processing via external driving fields nor the effect of electric field on the ground state properties has been addressed yet. The present study is a contribution to fill these gaps.

We note that the electric field coupling term resembles a Dzyaloshinskii-Morija (DM) anisotropy, with a coupling constant $d=g_{M E} E$. Experiments indicate the presence of a small DM anisotropy in MF cuprates made of frustrated spin chains [56,57]; previous theories considered it negligible, however. Here we show that even a tiny DM anisotropy modifies considerably the spin- $\frac{1}{2}$ chain characteristics. In particular, nematic spin-density-wave (SDW) state of magnon as well as multipolar phases transform into a chiral Luttinger liquid with nonzero spin current in the ground state.

\section{A. Minimal system of four spins}

First we focus analytically on a minimal system of four spins for different strengths of magnetic and electric (driving) fields for establishing an efficient protocol to field control the entanglement. We also inspect quantum state transfer fidelity (QSTF) through the MF chain and its $E$-field dependence.

For strong $B$ fields, i.e., $B$ is much larger than $\left|J_{1}\right|, 1$, and $d$ where

$$
d=g_{M E} E,
$$

the ground state is fully polarized state $|F\rangle=|\uparrow \uparrow \uparrow \uparrow\rangle$, with the corresponding energy,

$$
\mathcal{E}_{F}=J_{1}+1-2 B \text {. }
$$

The pair entanglement between any two arbitrary spins and the chirality vanish. Decreasing the magnetic field so that

$$
B_{0}<B<d+J_{1}+2,
$$

where $B_{0}=\sqrt{\left(J_{1}-4\right)^{2}+8 d^{2}} / 2+\left(J_{1}-2 d\right) / 2$, the ground state becomes

$$
\begin{aligned}
\left|\psi_{1}\right\rangle= & \frac{i}{2}|\downarrow \uparrow \uparrow \uparrow\rangle+\frac{-1}{2}|\uparrow \downarrow \uparrow \uparrow\rangle+\frac{-i}{2}|\uparrow \uparrow \downarrow \uparrow\rangle \\
& +\frac{1}{2}|\uparrow \uparrow \uparrow \downarrow\rangle
\end{aligned}
$$

with the corresponding energy

$$
\mathcal{E}_{1}=-1-B-d \text {. }
$$

The chirality jumps to

$$
\kappa=\left\langle\psi_{1}\left|\kappa_{i}\right| \psi_{1}\right\rangle=1 / 4 .
$$

We observe a finite entanglement, as quantified by the pair concurrence between spins on $n$ and $m$ sites [59]

$$
C_{n m}=\max \left(0, \sqrt{R_{1}}-\sqrt{R_{2}}-\sqrt{R_{3}}-\sqrt{R_{4}}\right),
$$

where $R_{n}$ are the eigenvalues of the matrix

$$
R=\rho_{n m}^{R}\left(\sigma_{1}^{y} \bigotimes \sigma_{2}^{y}\right)\left(\rho_{n m}^{R}\right)^{*}\left(\sigma_{1}^{y} \bigotimes \sigma_{2}^{y}\right),
$$

and $\rho_{n m}^{R}$ is the reduced density matrix of the four spins system obtained from the density matrix $\hat{\rho}$ after tracing over two spins. One can contrast the amount of the entanglement stored in the pair correlations, quantified by the so-called two-tangle $\tau_{2}$, with the multi-spin entanglement of the whole spin chain, encapsulated in the one tangle,

$$
\tau_{1}=4 \operatorname{det} \rho_{1},
$$

where $\rho_{1}$ is the single spin reduced density matrix [59]. The two tangle is calculated as

$$
\tau_{2}=\sum_{m}^{4} C_{n m}^{2} .
$$

For the state $\left|\psi_{1}\right\rangle$ we find the ratio $\tau=\frac{\tau_{2}}{\tau_{1}}=1$, thus half of the entanglement generated by decreasing the magnetic field (or increasing the electric field) in $\left|\psi_{1}\right\rangle$ is stored in the collective multi-spin correlations and half in the pair correlations. It is instructive to study the effect of $E$ and $B$ fields on QSTF [60] between different states,

$$
\begin{aligned}
F(E, B, t) & =\frac{\left|f_{j, s}(E, B, t)\right| \cos \gamma}{3}+\frac{\left|f_{j, s}(E, B, t)\right|^{2}}{6}+\frac{1}{2}, \\
\gamma & =\arg \left\{f_{j, s}(E, B, t)\right\} .
\end{aligned}
$$

Here

$$
f_{j, s}(E, B, t)=\langle j|\exp (-i \hat{H} t)| s\rangle
$$

is the transition amplitude between the states $|j\rangle$ and $|s\rangle$.

Time dependencies of QSTF obtained analytically between the initial state $|1\rangle=|\downarrow \uparrow \uparrow \uparrow\rangle$ and the final states $|2\rangle=$ $|\uparrow \downarrow \uparrow \uparrow \uparrow\rangle$ and $|3\rangle=|\uparrow \uparrow \downarrow \uparrow\rangle$ are depicted in Fig.1. Transition amplitudes and energy levels entering in the expression for fidelity Eq. (2) for these cases are:

$$
\begin{aligned}
f_{1,2}= & \frac{1}{4}\left(\exp \left[-i \wp_{5} t\right]-\exp \left[-i \wp_{4} t\right]\right) \\
& -\frac{i}{4}\left(\exp \left[-i \wp_{2} t\right]-\exp \left[-i \wp_{3} t\right]\right), \\
f_{2,1}= & -\frac{1}{4}\left(\exp \left[-i \wp_{4} t\right]-\exp \left[-i \wp_{5} t\right]\right) \\
& -\frac{i}{4}\left(\exp \left[-i \wp_{2} t\right]-\exp \left[-i \wp_{3} t\right]\right), \\
f_{1,3}= & f_{3,1}=-\frac{1}{4}\left(\exp \left[-i \wp_{2} t\right]+\exp \left[-i \wp_{3} t\right]\right. \\
& \left.-\exp \left[-i \wp_{4} t\right]-\exp \left[-i \wp_{5} t\right]\right),
\end{aligned}
$$

where

$$
\begin{aligned}
& \wp_{2}=-J_{2}-B-d, \quad \wp_{3}=-J_{2}-B+d, \\
& \wp_{4}=-J_{1}+J_{2}-B, \quad \wp_{5}=J_{1}+J_{2}-B .
\end{aligned}
$$

The results evidence that $E$ field increases QSTF, particularly from $|1\rangle$ to $|3\rangle$. By inspecting Eq. (2) we infer that the oscillating behavior of $F$ in Fig. 1 is related to the interference effect between different quantum states $\mathcal{E}_{n}(E) / \hbar$. Note that electric field $E$ enters in the energy levels through the DM coupling leading to a shift of energies of the states and to modification of transition strengths.

For confirmation we performed numerical calculations for systems with a large number of spins (not shown) and observed 


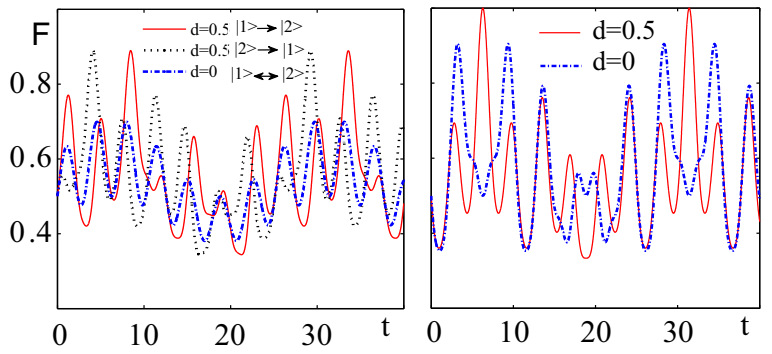

FIG. 1. (Color online) Time and $E$-field dependence of the quantum state transfer fidelity in a four spin chain, as quantified by $F$ [cf. Eq. (2)]. $d=g_{M E} E$ is measured in units of $J_{2}$. Left panel: $F$ for the transfer between states, $|1\rangle \rightarrow|2\rangle$ and $|2\rangle \rightarrow|1\rangle$. Right panel: $F$ for $|1\rangle \rightarrow|3\rangle=|3\rangle \rightarrow|1\rangle$. We set dimensionless units $J_{1} / J_{2}=-1, B / J_{2}=1 / 4$. Time is measured in $\hbar / J_{2}$. In material parameters, e.g., for $\mathrm{LiCu}_{2} \mathrm{O}_{2}$ (cf. Ref. [43]) with $\hbar / J_{2} \simeq 0.1$ [ps], assuming a unit cell of size $a_{F E} \approx 10[\mathrm{~nm}]$ and a polarization of $P=P_{0} a_{F E}^{3}$ with $P_{0}=5 \times 10^{-6}\left[\mathrm{C} / \mathrm{m}^{2}\right]$ (which is within the range measured in Ref. [29]), $d=0.5$ corresponds to $E=10^{3}[\mathrm{kV} / \mathrm{cm}]$. As we choose $\mathbf{S}_{N+1}=\mathbf{S}_{1}$, for $N=4$ the transition $|1\rangle \rightarrow|3\rangle$ shows no directional dependence for the fidelity.

similar behavior of QSTF on $E$. We note that $E$ field breaks the parity symmetry of the MF spin chain. Hence, when $E$ field is present, clockwise and anticlockwise QSTF between the states $|j\rangle \longrightarrow|s\rangle$ and $|s\rangle \longrightarrow|j\rangle$ differ considerably as depicted in Fig. 1, which might be used for information transfer control via electric field [61]. Further decreasing the magnetic field below $B_{0}$, the ground state becomes

$$
\begin{aligned}
\left|\psi_{2}\right\rangle= & \beta(|\uparrow \uparrow \downarrow \downarrow\rangle-i \lambda|\downarrow \uparrow \downarrow \uparrow\rangle-|\uparrow \downarrow \downarrow \uparrow\rangle-|\downarrow \uparrow \uparrow \downarrow\rangle \\
& +i \lambda|\uparrow \downarrow \uparrow \downarrow\rangle+|\downarrow \downarrow \uparrow \uparrow\rangle),
\end{aligned}
$$

where

$$
\lambda=\frac{J_{1} / 2-2+2 \sqrt{1+J_{1}^{2} / 16-J_{1} / 2+d^{2} / 2}}{d}
$$

and $\beta=1 / \sqrt{4+2 \lambda^{2}}$. In this case for chirality we have

$$
\kappa=\left\langle\psi_{2}\left|\kappa_{i}\right| \psi_{2}\right\rangle=8 \lambda \beta^{2} .
$$

Its electric field dependence is shown in Fig. 2(a). The ratio between one-tangle $\tau_{1}$ and two-tangle $\tau_{2}$ in the ground state
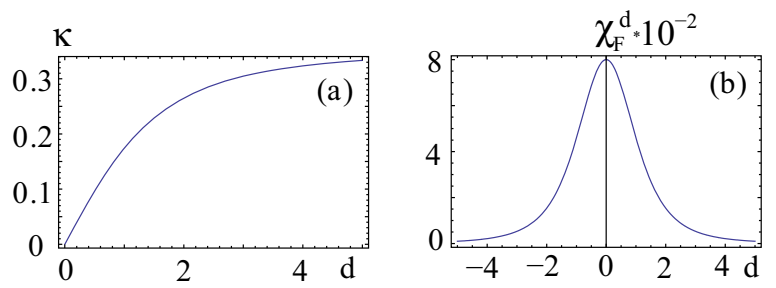

FIG. 2. (Color online) (a) Electric field dependence of chirality $\kappa$ for the following values of the parameters: $-J_{1}=J_{2}=1, B=1 / 4$. We see that electric field generates chirality. Qualitatively similar dependence holds even in thermodynamic limit. Electric field control of the magnetic chirality in the ferroaxial MF system $\mathrm{RbFe}\left(\mathrm{MoO}_{4}\right)_{2}$ was addressed in Ref. [63]. (b) Electric field fidelity susceptibility. Even a weak electric field leads to a substantial reduction of the FS. $\left|\psi_{2}\right\rangle$ reads

$$
\tau=\frac{\tau_{2}}{\tau_{1}}=\left(\frac{2-\lambda^{2}}{2+\lambda^{2}}\right)^{2}<1,
$$

for $0<d \leqslant\left(4-J_{1}\right) / 14$. Therefore, in this case the entanglement generated by the electric field is stored basically in many spin correlations rather than in two spin correlations.

The response sensitivity to changing the driving field amplitude is quantified by the fidelity susceptibility (FS) [62] FS with respect to magnetic field vanishes as the magnetization is conserved in our model. FS with $E$-field changes is finite. E.g., for $\left|\psi_{2}\right\rangle$ state we obtain

$$
\chi_{F}^{d}=(\alpha \beta / d)^{2}
$$

and depict it in Fig. 2(b). As we see even small amplitude of the electric field leads to the substantial reduction of the FS. We will study FS for longer chains later, especially its behavior near the nematic to chiral quantum phase transition (QPT). Hence depending on the driving fields, quantum information characteristics such as many particle entanglement and QSTF differ considerably.

\section{B. Thermodynamic limit}

For a macroscopic number of sites, in the MF chain, we can expect that different quantum phases and QPTs can be controlled by $E$ field. We study this below. We focus on the thermodynamic limit. Before addressing the many-body physics it is instructive to start with the two-magnon problem: For $d=0$ and a weak $J_{1}<0$ a bound state of two magnons forms below the scattering continuum. The bound state branch has a minimum for the total momentum $P=\pi$, because antiferromagnetic $J_{2}$ disfavors two magnons occupying sites of the same parity. We solved analytically the two-magnon problem for $d \neq 0$ (for $L \rightarrow \infty$ ). The two-magnon spectrum with scattering states, important for the low energy bound state branch, is depicted in Fig. 3. The main result is that with including $d \neq 0$, the bound state minimum of the two-magnon state shifts immediately from $P=\pi$ to $P=\pi-P_{0}$, where $P_{0} \sim d$. The binding energy decreases as well, gradually with increasing $d$, and after a critical value of $d>d_{c}\left(J_{1}\right)$ (e.g., for $J_{1}=-1, d_{c} \simeq 0.183$ ) the two-magnon scattering state

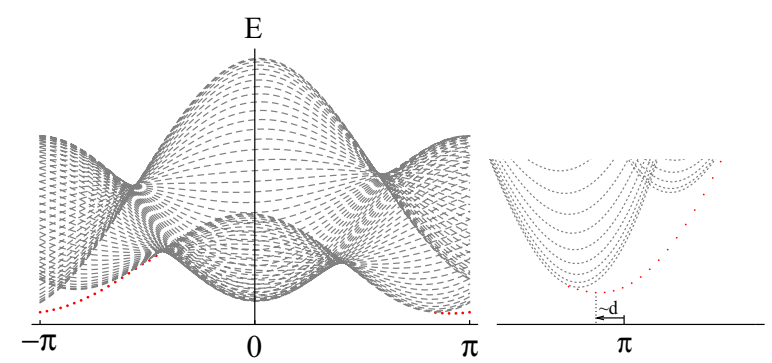

FIG. 3. (Color online) Left panel: two-magnon spectrum, with scattering states and low energy bound state branch for $d=0.15$ and $J_{1}=-1$. Parity asymmetry of the spectrum is due to DzyaloshiskiiMoriya anisotropy $d$. Right panel: a zoom of the two-body dispersion around the momentum $\pi$ ( $\pi$ and $-\pi$ are equivalent points) indicating a shift of the minimum from $\pi$ in the direction of the minimum of two-magnon scattering states. 


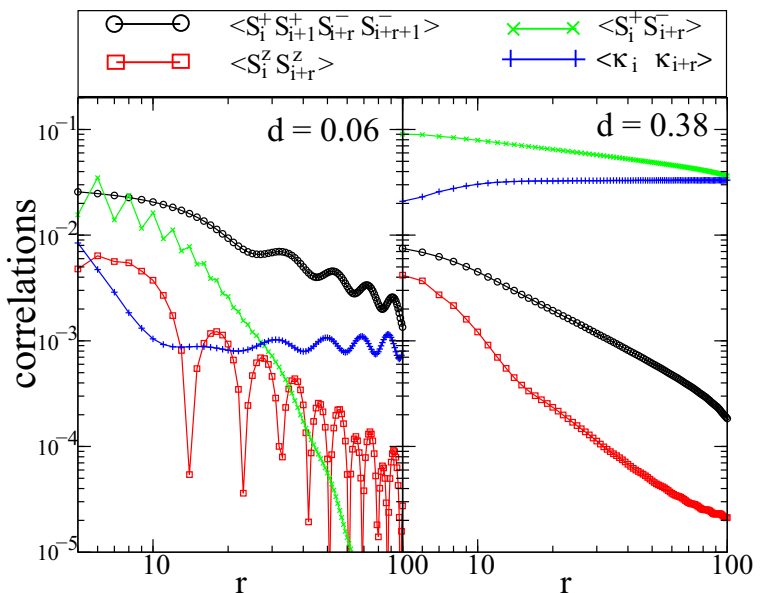

FIG. 4. (Color online) Various correlation functions for $J_{1}=-1$ and $M=0.4$ in nematic (left panel) and chiral (right panel) phases for $L=160$ sites. In-plane spin-spin correlation functions $\left\langle S_{i}^{+} S_{i+r}^{-}\right\rangle$show exponential decay in nematic phase and algebraic quasi-long-range order in the chiral phase. Pair correlations $\left\langle S_{i}^{+} S_{i+1}^{+} S_{i+r}^{-} S_{i+r+1}^{-}\right\rangle$and the density correlations $\left\langle S_{i}^{z} S_{i+r}^{z}\right\rangle$ decay algebraically in both phases with pronounced oscillations in nematic phase. Chirality-chirality correlation function $\left\langle\kappa_{i} \kappa_{i+r}\right\rangle$ also show oscillations in nematic phase due to open boundary conditions.

minimum becomes energetically lower and the two-magnon bound states disappear from the ground state.

When the density of magnons is increased with decreasing of the magnetic field, we expect that the two-magnon bound states quasicondense in the minimum of the two-magnon dispersion at $P=\pi-P_{0}$. Hence, the ground state will enter the nematic-chiral state for an arbitrary small $d \neq 0$. However, when $d>d_{c}$, the nematicity (magnon pair quasicondensate) disappears via QPT, and the low energy behavior is dominated by a single-particle picture with $\left\langle S_{i}^{-} S_{j}^{+}\right\rangle$quasi-long-range ordered as shown in the right panel of Fig. 4. Hence, we anticipate an $E$-field driven phase transition from the "molecular" (two-magnon bound state) quasicondensate to the "atomic" (single-particle) quasicondensate. This expectation is fully confirmed by the effective field theory description within the bosonization techniques (see Appendix A) where the competition between ferromagnetic $J_{1}$ (that binds magnons and produces nematic order) and electric field (promoting chirality) is resolved via an Ising QPT with changing $d$.

\section{NUMERICAL SIMULATIONS}

We have checked our analytical results with large scale numerical calculations using the density matrix renormalization group method $[64,65]$ implemented for the open boundary conditions.

For small values of $\left|J_{1}\right| \lesssim 2$ and for $d=0$ the leading correlation function is $\left\langle S_{i}^{z} S_{j}^{z}\right\rangle$ for low magnetic fields $B \neq 0$ and the system is in the SDW dominated regime. With increasing the magnetic field SDW phase crosses over into the nematic state [52], with leading correlation function given by $\left\langle S_{i}^{-} S_{i+1}^{-} S_{j}^{+} S_{j+1}^{+}\right\rangle$(see left panel of Fig. 4). In both regimes the in-plane single-spin correlation function $\left\langle S_{i}^{-} S_{j}^{+}\right\rangle$decays
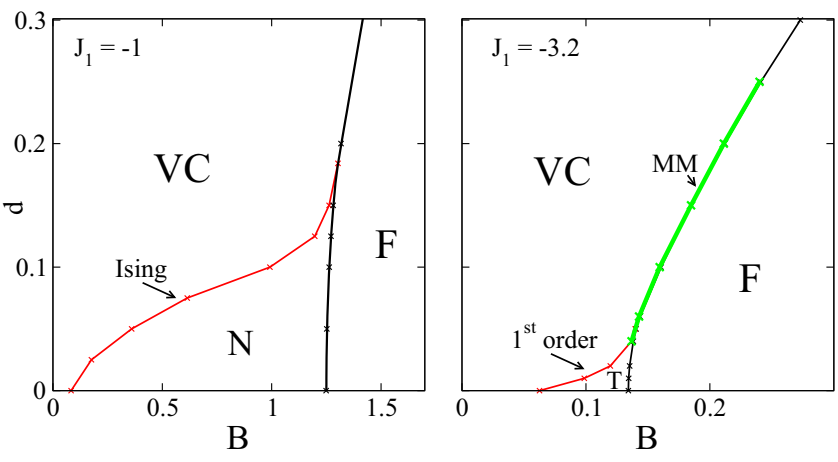

FIG. 5. (Color online) Phase diagram as a function of electric and magnetic fields for different values of $J_{1}$. MM indicates metamagnetic behavior (macroscopic jump) in the magnetization when descending from a saturation value. T indicates a multipolar state with three-body bound states. We determined phase boundary between nematic $(\mathrm{N})$ and chiral (VC) states by looking at magnetization step size with $B$ for finite systems. $\Delta M=2$ in $\mathrm{N}$, whereas $\Delta M=1$ in VC. Similarly, we observe the phase boundary between $\mathrm{T}$ (with magnetization step $\Delta M=3)$ and a VC.

exponentially. We have studied various correlation functions for different values of electric field. In Fig. 4 we compare the behavior of the correlation functions in nematic $\left(d<d_{c}\right)$ and chiral $\left(d>d_{c}\right)$ phases. In Fig. 5 we depict the phase diagram as a function of driving fields $E$ and $B$ at $J_{1}=-1$ (left panel) and $J_{1}=-3.2$ (right panel). We determined the phase boundary between nematic (N) and chiral (VC) states by looking at magnetization step size with $B$ for finite systems. $\Delta M=2$ in $\mathrm{N}$, whereas $\Delta M=1$ in $\mathrm{VC}$ as depicted in the left panel of Fig. 6. Similarly, we observe the phase boundary between $\mathrm{T}$ (with magnetization step $\Delta M=3$ ) and VC phases as depicted in the right panel of Fig. 6. In contrast to the smooth behavior of the magnetization curve at the transition from $\mathrm{VC}$ into $\mathrm{N}$ states with changing $d$, at the transition from $\mathrm{VC}$ to $\mathrm{T}$ state magnetization experiences a macroscopic jump rendering this transition first order.
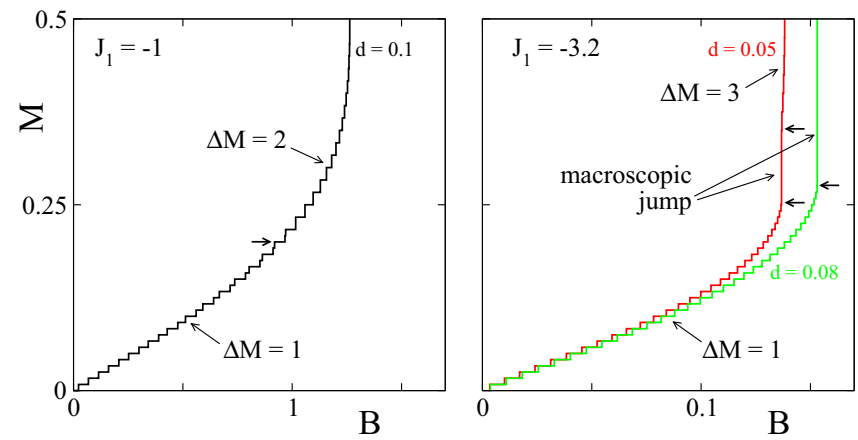

FIG. 6. (Color online) Magnetization curves of the finite chain of $L=120$ sites for different values of $d$ and $J_{1}$. Left panel: magnetization curve showing transition from VC (magnetization step size $\Delta M=1$ ) in $\mathrm{N}$ phase (magnetization step size $\Delta M=2$ ). Right panel: magnetization curves showing transition either from VC into $\mathrm{T}$ (magnetization step size $\Delta M=3$ ) via magnetization jump, or for stronger DM coupling from VC directly MM transition into the fully polarized state. 

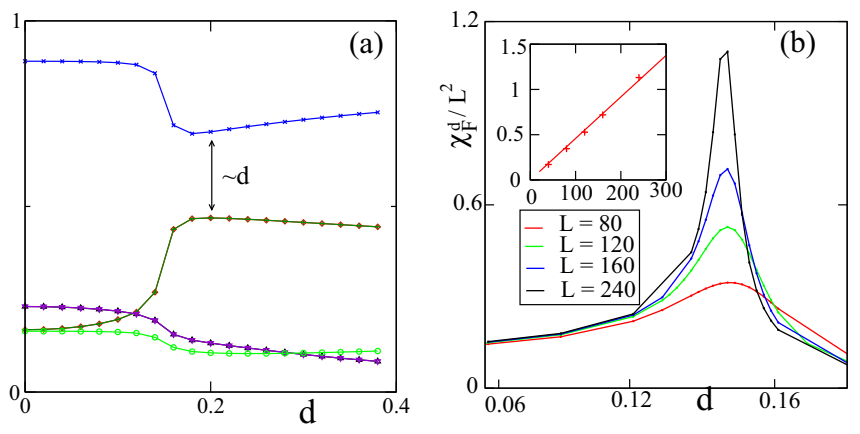

FIG. 7. (Color online) (a) Entanglement spectrum (only four highest eigenvalues of the reduced density matrix are shown) for $L=160$ sites. (b) Scaling of DM fidelity susceptibility near the nematic to chiral QPT for $J_{1}=-1$ and $M=0.4$.

To witness the transition from the nematic to the chiral state induced by $E$ we studied the behavior of the entanglement spectrum [Fig. 7(a)] and DM fidelity susceptibility [Fig. 7(a)]. In the chiral phase of a $J_{1}-J_{2}$ chain and for $d=0$ the complete entanglement spectrum is doubly degenerate due to the spontaneously broken parity symmetry, however in the presence of $d$ the degeneracy is lifted. Linear in $L$ scaling of the peak of DM fidelity susceptibility relative to the overall background shown in the inset of Fig. 7(b) confirms the Ising nature of QPT.

We have studied as well the effect of DM anisotropy on multipolar phases of the $J_{1}-J_{2}$ chain for $-4<J_{1}<-2.7$ involving bound states with more than two magnons. The minimum of the multi-body bound state dispersion which is at $K=\pi$ for $d=0$ (in both phases $\mathrm{T}$ and $\mathrm{Q}$ ) shifts from $\pi$ immediately for $d \neq 0$. In fact, $1 \% \sim 2 \% \mathrm{DM}$ anisotropy in the nearest neighbor exchange interaction $J_{1}$ is sufficient to

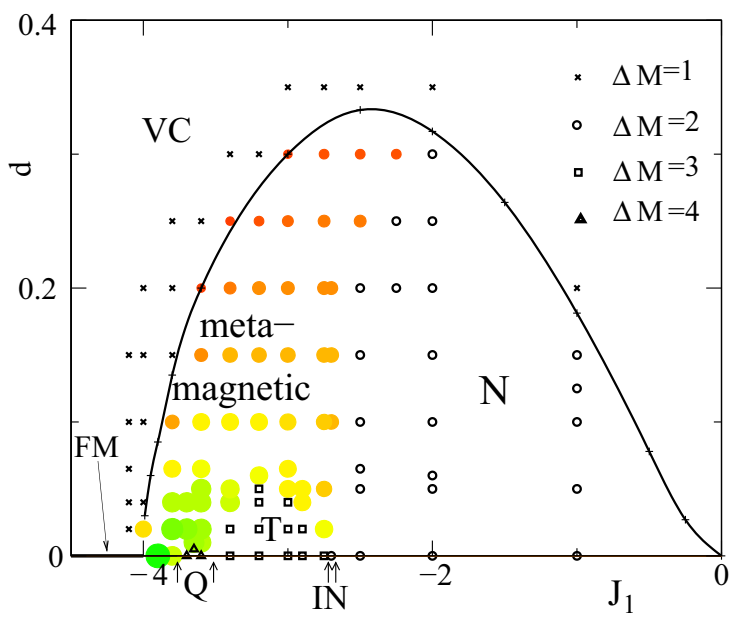

FIG. 8. (Color online) Phases under saturation magnetization. N, T, and Q stand for multipolar phases with two-, three-, and fourmagnon bound states, respectively, and IN stands for incommensurate nematic phase. Filled circles indicate that for these parameters the system experiences a macroscopic magnetization jump when descending from a fully polarized ground state into VC state by lowering $B$ (indicated by $\mathrm{MM}$ in Fig. 5), a larger circle meaning a greater jump. remove the three-body and the four-body multipolar phases from the ground state phase diagram below the saturation magnetization. Instead, in the presence of a tiny $d \neq 0$ the ground state magnetization experiences a macroscopic jump to the fully saturated value when increasing the magnetic field as depicted in Fig. 8. Note, for $d=0$ the metamagnetic region is squeezed in the close right-side vicinity of $J_{1}=-4$ point. In the presence of DM anisotropy the metamagnetic jump is observed in a much broader region, starting at $J_{1} \simeq-2$ and extending even in the region $J_{1}<-4$ (see Appendix B).

\section{CONCLUSION}

In summary, based on the spin current model for a helical multiferroic spin- $\frac{1}{2}$ chain in external $B$ and $E$ fields we found that both quantum information processing as well as ground state phases are extremely sensitive to an electric field that affects the magnetoelectric coupling. $E$-field increases strongly the quantum state transfer fidelity and makes it directional dependent (transfer in clockwise direction differs from that in anticlockwise direction). A tiny magnetoelectric coupling is sufficient to change the spin-density-wave/nematic or multipolar phases in favor of the chiral phase. We analyzed QPT induced by ME coupling and found in particular a sharp change of the entanglement spectrum and a large enhancement of the fidelity susceptibility at Ising QPT from nematic to chiral states. Our findings serve as the basis for $E$ field controlled quantum information processing in helical multiferroics.

\section{ACKNOWLEDGMENTS}

M.A., L.C., S.K.M., and J.B. gratefully acknowledge financial support by the Deutsche Forschungsgemeinschaft (DFG) through SFB 762, and contract BE 2161/5-1. S.G. and T.V. are supported by QUEST (Center for Quantum Engineering and Space-Time Research) and DFG Research Training Group (Graduiertenkolleg) 1729.

\section{APPENDIX A: DETAILS OF BOSONIZATION}

Here we provide details of effective field theory description, bosonization applied to microscopic Hamiltonian Eq. (1). To develop bosonization description it is convenient to consider the limit of strong frustration $J_{2} \gg\left|J_{1}\right|$ and weak DM anisotropy $d \ll J_{2}$. In this case the system may be viewed as two antiferromagnetic spin- $\frac{1}{2}$ chains weakly coupled by the zigzag interchain coupling $J_{1}$ [66] with DM anisotropy $d$.

Low-energy properties of a single spin- $\frac{1}{2}$ chain in a uniform magnetic field is described by the standard Gaussian theory [67] known also as the Tomonaga-Luttinger liquid:

$$
\mathcal{H}=\frac{v}{2} \int d x\left\{\frac{1}{K}\left(\partial_{x} \phi\right)^{2}+K\left(\partial_{x} \theta\right)^{2}\right\} .
$$

Here $\phi$ is a real scalar bosonic field and $\theta$ is its dual field, $\partial_{t} \phi=$ $v \partial_{x} \theta$, with the commutation relations $[\phi(x), \theta(y)]=i \Theta(y-$ $x$ ), where $\Theta(x)$ is the Heaviside function. $K$ is Luttinger liquid parameter and $v$ is spin-wave velocity.

The exact functional dependencies $v\left(J_{2}, B\right)$ and $K\left(J_{2}, B\right)$ for isolated chains are known (see [68] and references therein) 
from the numerical solution of the Bethe ansatz integral equations [69]. In particular, $K$ increases monotonously with the magnetic field, whereas $v$ decreases: $K(B=0)=\frac{1}{2}$, $v(B=0)=J_{2} \pi / 2$ and $K \rightarrow 1, v \rightarrow 0$ for $B \rightarrow B_{\text {sat }}$, where saturation value $B_{\text {sat }}=2 J_{2}$.

Long wavelength fluctuations of a spin- $\frac{1}{2}$ chain are captured by the following representation of the lattice spin operators [67]:

$$
\begin{gathered}
S_{n}^{z} \rightarrow \frac{1}{\sqrt{\pi}} \partial_{x} \phi+\frac{a}{\pi} \sin \left\{2 k_{F} x+\sqrt{4 \pi} \phi\right\}+M \\
S_{n}^{-} \rightarrow(-1)^{n} e^{-i \theta \sqrt{\pi}}\left\{c+b \sin \left(2 k_{F} x+\sqrt{4 \pi} \phi\right)\right\} .
\end{gathered}
$$

Here $M(B)$ is the ground state magnetization per spin which determines the Fermi wave vector $k_{F}=\left(\frac{1}{2}-M\right) \pi$ and $a, b$, and $c$ are nonuniversal numerical constants.

For $J_{1}=d=0$, two decoupled chains are described by two copies of Gaussian models of the form (A1) with a pair of dual bosonic fields $\left[\phi_{1}, \theta_{1}\right]$ and $\left[\phi_{2}, \theta_{2}\right]$. Treating interchain couplings $J_{1}$ and DM anisotropy $d$ perturbatively and introducing the symmetric and antisymmetric combinations of the fields describing the individual chains, $\phi_{ \pm}=\left(\phi_{1} \pm \phi_{2}\right) / \sqrt{2 K}$ and $\theta_{ \pm}=\left(\theta_{1} \pm \theta_{2}\right) \sqrt{K / 2}$, the effective Hamiltonian density describing low-energy properties of the microscopic model given in Eq. (1) takes the following form:

$$
\begin{aligned}
\mathcal{H}_{\text {eff }}= & \mathcal{H}_{0}^{+}+\mathcal{H}_{0}^{-}+\mathcal{H}_{\text {int }}, \\
\mathcal{H}_{0}^{ \pm}= & \frac{v_{ \pm}}{2}\left[\left(\partial_{x} \theta_{ \pm}\right)^{2}+\left(\partial_{x} \phi_{ \pm}\right)^{2}\right], \\
\mathcal{H}_{\text {int }}= & g_{1} \cos \left(k_{F}+\sqrt{8 \pi K_{-}} \phi_{-}\right) \\
& -\left(g_{2} \partial_{x} \theta_{+}+g_{3}\right) \sin \left(\sqrt{2 \pi / K_{-}} \theta_{-}\right) .
\end{aligned}
$$

The Fermi velocities $v_{ \pm} \propto J_{2}$ and coupling constants are $g_{1} \propto J_{1} \cos k_{F}$ [70], $g_{2} \propto J_{1}$, and $g_{3} \propto d$, with proportionality coefficients involving short-distance cutoff. The Luttinger liquid parameter of antisymmetric sector is given by

$$
K_{-}=K(B)\left\{1+\frac{J_{1} K(B)}{\pi v(B)}\right\} .
$$

The intersector coupling in Eq. (A3) contains a term with coupling constant $g_{2}$ that represents an infrared limit of the product of $z$ components of in-chain and interchain vector chiralities [71],

$$
\left(\kappa_{2 i-1,2 i+1}^{z}+\kappa_{2 i, 2 i+2}^{z}\right) \kappa_{2 i, 2 i+1}^{z} \rightarrow \partial_{x} \theta_{+} \sin \sqrt{\frac{2 \pi}{K_{-}}} \theta_{-},
$$

where $\kappa_{i, j}^{z} \equiv\left(\mathbf{S}_{i} \times \mathbf{S}_{j}\right)^{z}$

The Hamiltonian (A3) provides with the effective field theory describing the low-energy behavior of a strongly frustrated spin- $\frac{1}{2}$ zigzag chain with DM anisotropy for a nonzero magnetization $M$. For small values of magnetization the Luttinger liquid parameter $K_{-} \simeq \frac{1}{2}$, and the intersector $g_{2}$ term has a higher scaling dimension than the strongly relevant $g_{1}$ and $g_{3}$ terms in the antisymmetric sector. In this case the system is in a phase with relevant competing couplings in antisymmetric sector. In contrast to that, at $B=0$ all terms generated by the $J_{1}$ zigzag coupling are marginal and only DM coupling $g_{3}$ is a relevant perturbation. The competition between $\cos \sqrt{8 \pi K_{-}} \phi_{-}$(nematicity) and $\cos \sqrt{2 \pi / K_{-}} \theta_{-}$(chirality) terms is resolved with an Ising phase transition in the antisymmetric sector with changing $d / J_{1}[72]$.

\section{APPENDIX B: EFFECT OF DM ANISOTROPY IN FERROMAGNETIC REGION $J_{1}<-4 J_{2}$}

We now discuss the effect of DM interaction on ferromagnetic region $J_{1}<-4 J_{2}$. For $d=0$, due to $\mathrm{SU}(2)$ symmetry the magnon gas behaves as noninteracting bosons. Deep inside ferromagnetic region DM interaction introduces repulsion (repulsion increases monotonously with increasing $d$ ) between magnons and below the fully polarized state chiral Luttinger liquid phase is realized for any $d \neq 0$ [73]. However, in close left-side vicinity of $J_{1}=-4$ (hence $J_{1}<-4$ ) the nonmonotonous effect of DM on the effective interaction between magnons is observed. First, for small values $d \rightarrow$ 0 DM anisotropy introduces repulsion between magnons, however with increasing $d$ repulsion transforms into attraction and with further increasing $d$ interaction between magnons becomes repulsive once again as shown in Fig. 1. Effective coupling constant of the magnon gas we extracted from the following relation $[74,75]$,

$$
g=-\frac{2 \hbar^{2}}{m a_{1 D}}
$$

where $m$ is mass of magnon and $a_{1 D}$ is one-dimensional scattering length, which we calculated analytically from the low energy scattering phase shift $\delta(k)$,

$$
a_{1 D}=\lim _{k \rightarrow 0} \frac{\delta(k)}{k},
$$

where $k$ is a relative momentum of scattering magnons.

For the attractive regime $g<0, a_{1 D}>0$, the scattering length extracted from the scattering problem coincides with the correlation length of the bound state of magnons. We depict in Fig. 9 the scattering length from which one can observe, due to Eq. (B1), that effective interaction changes sign twice via resonancelike behavior when changing $d$. For the values of $d$ which correspond to the positive scattering length (and hence $g<0$ ), the external magnetic field induces a metamagnetic transition (macroscopic jump of the magnetization) from chiral Luttinger liquid to the fully polarized state (resulting in first

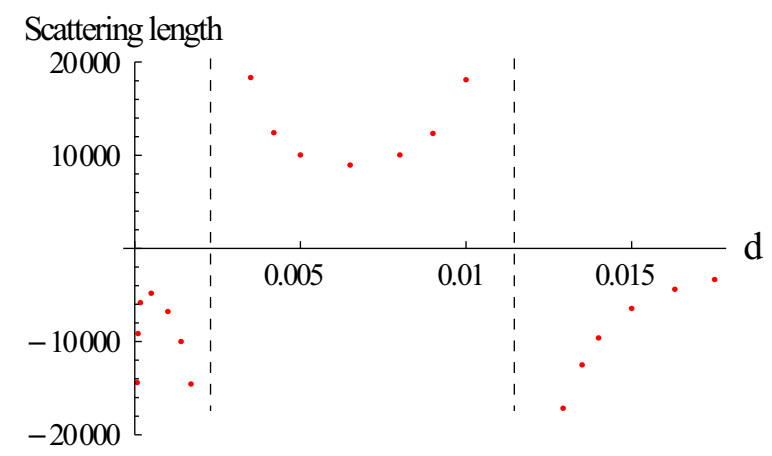

FIG. 9. (Color online) Two-magnon scattering length in units of the lattice constant for $J_{1}=-4.005$, showing nontrivial sequence of resonances induced by changing just a single parameter $d$. 
order phase transition). For the parameters corresponding to negative scattering length (and hence $g>0$ ) magnetization will change smoothly all the way from $M=0$ until $M=1 / 2$, in particular leading to usual commensurate-incommensurate phase transition from chiral Luttinger liquid to fully polarized state when increasing the magnetic field strength.
[1] H. Schmid, Int. J. Magn. 4, 337 (1973); Ferroelectrics 162, 317 (1994); J. Phys.: Condens. Matter 20, 434201 (2008).

[2] W. Eerenstein, N. D. Mathur, and J. F. Scott, Nature (London) 442, 759 (2006).

[3] Y. Tokura and S. Seki, Adv. Mater. 22, 1554 (2010).

[4] C. A. F. Vaz, J. Hoffman, Ch. H. Ahn, and R. Ramesh, Adv. Mater. 22, 2900 (2010).

[5] F. Zavaliche, T. Zhao, H. Zheng, F. Straub, M. P. Cruz, P. L. Yang, D. Hao, and R. Ramesh, Nano Lett. 7, 1586 (2007).

[6] H. L. Meyerheim, F. Klimenta, A. Ernst, K. Mohseni, S. Ostanin, M. Fechner, S. Parihar, I. V. Maznichenko, I. Mertig, and J. Kirschner, Phys. Rev. Lett. 106, 087203 (2011).

[7] J. Wang, J. B. Neaton, H. Zheng, V. Nagarajan, S. B. Ogale, B. Liu, D. Viehland, V. Vaithyanathan, D. G. Schlom, U. V. Waghmare, N. A. Spaldin. K. M. Rabe, M. Wuttig, and R. Ramesh, Science 299, 1719 (2003).

[8] R. Ramesh and N. A. Spaldin, Nat. Mater. 6, 21 (2007).

[9] V. Garcia, M. Bibes, L. Bocher, S. Valencia, F. Kronast, A. Crassous, X. Moya, S. Enouz-Vedrenne, A. Gloter, D. Imhoff, C. Deranlot, N. D. Mathur, S. Fusil, K. Bouzehouane, and A. Barthélémy, Science 327, 1106 (2010).

[10] M. Bibes and A. Barthelemy, Nat. Mater. 7, 425 (2008).

[11] N. Kida and Y. Tokura, J. Magn. Magn. Mat. 324, 3512 (2012); M. I. Belinsky, Phys. Rev. B 84, 064425 (2011); D. I. Khomskii, J. Phys.: Condens. Matter 22, 164209 (2010).

[12] S. Picozzia and A. Stroppa, Eur. Phys. J. B 85, 240 (2012).

[13] M. Dawber, K. M. Rube, and J. F. Scott, Rev. Mod. Phys. 77, 1083 (2005).

[14] S. Valencia, A. Crassous, L. Bocher, V. Garcia, X. Moya, R. O. Cherifi, C. Deranlot, K. Bouzehouane, S. Fusil, A. Zobelli, A. Gloter, N. D. Mathur, A. Gaupp, R. Abrudan, F. Radu, A. Barthélémy, and M. Bibes, Nat. Mater. 10, 753 (2011).

[15] M. Fiebig, J. Phys. D 38, R123 (2005).

[16] S. W. Cheong and M. Mostovoy, Nat. Mater. 6, 13 (2007).

[17] W. Prellier, M. P. Singh, and P. Murugavel, J. Phys. 17, R803 (2005).

[18] Y. Tokura, J. Magn. Magn. Mat. 310, 1145 (2007).

[19] C. N. R. Rao and C. R. Serrano, J. Mat. Chem. 17, 4931 (2007).

[20] G. Lawes and G. Srinivasan, J. Phys. D 44, 243001 (2011).

[21] J. van den Brink and D. I. Khomskii, J. Phys. 20, 434217 (2008).

[22] L. Chotorlishvili, R. Khomeriki, A. Sukhov, and S. Ruffo, and J. Berakdar, Phys. Rev. Lett. 111, 117202 (2013).

[23] M. Gajek, M. Bibe, S. Fusil, K. Bouzehouane, J. Fontcuberta, A. Barthélémy, and A. Fert, Nat. Mater. 6, 296 (2007).

[24] D. Pantel, S. Goetze, D. Hesse, and M. Alexe, Nat. Mater. 11, 289 (2012)

[25] C. L. Jia, and J. Berakdar, Appl. Phys. Lett. 95, 012105 (2009); 98, 192111 (2011); 98, 042110 (2011); 103, 052903 (2013).

[26] K. Taniguchi, N. Abe, T. Takenobu, Y. Iwasa, and T. Arima, Phys. Rev. Lett. 97, 097203 (2006).

[27] D. Higashiyama, S. Miyasaka, N. Kida, T. Arima, and Y. Tokura, Phys. Rev. B 70, 174405 (2004)

[28] N. Hur, S. Park, P. A. Sharma, J. S. Ahn, S. Guha, and S. W. Cheong, Nature (London) 429, 392 (2004).
[29] S. Seki, Y. Yamasaki, M. Soda, M. Matsuura, K. Hirota, and Y. Tokura, Phys. Rev. Lett. 100, 127201 (2008).

[30] H. Sagayama, K. Taniguchi, N. Abe, T. H. Arima, M. Soda, M. Matsuura, K. Hirota, Phys. Rev. B 77, 220407 (2008).

[31] A. Glavic, C. Becher, J. Voigt, E. Schierle, E. Weschke, M. Fiebig, and T. Brückel, Phys. Rev. B 88, 054401 (2013).

[32] T. Goto, T. Kimura, G. Lawes, A. P. Ramirez, Y. Tokura, Phys. Rev. Lett. 92, 257201 (2004); M. Kenzelmann, A. B. Harris, S. Jonas, C. Broholm, J. Schefer, S. B. Kim, C. L. Zhang, S. W. Cheong, O. P. Vajk, and J. W. Lynn, ibid. 95, 087206 (2005); Y. Yamasaki, S. Miyasaka, Y. Kaneko, J. P. He, T. Arima, and Y. Tokura, ibid. 96, 207204 (2006); J. Hemberger, F. Schrettle, A. Pimenov, P. Lunkenheimer, V. Y. Ivanov, A. A. Mukhin, A. M. Balbashov, and A. Loidl, Phys. Rev. B 75, 035118 (2007).

[33] I. E. Dzyaloshinkskii, Sov. Phys. JETP 10, 628 (1959); T. Moriya, Phys. Rev. 120, 91 (1960).

[34] C. Jia, S. Onoda, N. Nagaosa, and J. H. Han, Phys. Rev. B 76, 144424 (2007); S. Furukawa, M. Sato, and S. Onoda, Phys. Rev. Lett. 105, 257205 (2010).

[35] H. Katsura, N. Nagaosa, and A. V. Balatsky, Phys. Rev. Lett. 95 , 057205 (2005); I. A. Sergienko and E. Dagotto, Phys. Rev. B 73, 094434 (2006); M. Mostovoy, Phys. Rev. Lett. 96, 067601 (2006).

[36] Y. Yamasaki, H. Sagayama, T. Goto, M. Matsuura, K. Hirota, T. Arima, and Y. Tokura, Phys. Rev. Lett. 98, 147204 (2007).

[37] A. Pimenov, T. Rudolf, F. Mayr, A. Loidl, A. A. Mukhin, and A. M. Balbashov, Phys. Rev. B 74, 100403(R) (2006).

[38] A. Pimenov, A. A. Mukhin, V. Yu. Ivanov, V. D. Travkin, A. M. Balbashov, and A. Loidl, Nat. Phys. 2, 97 (2006).

[39] R. ValdesAguilar, A. B. Sushkov, C. L. Zhang, Y. J. Choi, S. W. Cheong, and H. D. Drew, Phys. Rev. B 76, 060404(R) (2007).

[40] A. Pimenov, A. Loidl, A. A. Mukhin, V. D. Travkin, V. Yu. Ivanov, and A. M. Balbashov, Phys. Rev. B 77, 014438 (2008).

[41] I. E. Chupis, Low. Temp. Phys. 33, 715 (2007); A. Cano and E. I. Kats, Phys. Rev. B 78, 012104 (2008).

[42] C. Jia and J. Berakdar, Eur. Phys. Lett. 88, 57004 (2009); Phys. Stat. Sol. B 247, 662 (2010).

[43] S. Park, Y. J. Choi, C. L. Zhang, and S-W. Cheong, Phys. Rev. Lett. 98, 057601 (2007).

[44] F. Schrettle, S. Krohns, P. Lunkenheimer, J. Hemberger, N. Büttgen, H.-A. Krug von Nidda, A. V. Prokofiev, and A. Loidl, Phys. Rev. B 77, 144101 (2008).

[45] T. Masuda, A. Zheludev, A. Bush, M. Markina, and A. Vasiliev, Phys. Rev. Lett. 92, 177201 (2004); T. Masuda, A. Zheludev, B. Roessli, A. Bush, M. Markina, and A. Vasiliev, Phys. Rev. B 72, 014405 (2005).

[46] A. A. Gippius, E. N. Morozova, A. S. Moskvin, A. V. Zalessky, A. A. Bush, M. Baenitz, H. Rosner, and S. L. Drechsler, Phys. Rev. B 70, 020406(R) (2004); S. L. Drechsler, J. Malek, J. Richter, A. S. Moskvin, A. A. Gippius, and H. Rosner, Phys. Rev. Lett. 94, 039705 (2005).

[47] M. Papagno, D. Pacile, G. Caimi, H. Berger, L. Degiorgi, and M. Grioni, Phys. Rev. B 73, 115120 (2006). 
[48] L. Mihaly, B. Dora, A. Vanyolos, H. Berger, and L. Forro, Phys. Rev. Lett. 97, 067206 (2006).

[49] A. V. Chubukov, Phys. Rev. B 44, 4693 (1991).

[50] A. Kolezhuk and T. Vekua, Phys. Rev. B 72, 094424 (2005).

[51] F. Heidrich-Meisner, A. Honecker, and T. Vekua, Phys. Rev. B 74, 020403(R) (2006).

[52] T. Vekua, A. Honecker, H.-J. Mikeska, and F. Heidrich-Meisner, Phys. Rev. B 76, 174420 (2007).

[53] L. Kecke, T. Momoi, and A. Furusaki, Phys. Rev. B 76, 060407(R) (2007).

[54] T. Hikihara, L. Kecke, T. Momoi, and A. Furusaki, Phys. Rev. B 78, 144404 (2008).

[55] J. Sudan, A. Lüscher, and A. M. Läuchli, Phys. Rev. B 80, 140402(R) (2009).

[56] M. Enderle, C. Mukherjee, B. Fåk, R. K. Kremer, J.-M. Broto, H. Rosner, S.-L. Drechsler, J. Richter, J. Malek, A. Prokofiev, W. Assmus, S. Pujol, J.-L. Raggazzoni, H. Rakoto, M. Rheinstädter, and H. M. Rønnow, Europhys. Lett. 70, 237 (2005).

[57] M. Enderle, B. Fak, H.-J. Mikeska, R. K. Kremer, A. Prokofiev, and W. Assmus, Phys. Rev. Lett. 104, 237207 (2010).

[58] S.-L. Drechsler, O. Volkova, A. N. Vasiliev, N. Tristan, J. Richter, M. Schmitt, H. Rosner, J. Malek, R. Klingeler, A. A. Zvyagin, and B. Büchner, Phys. Rev. Lett. 98, 077202 (2007).

[59] L. Amico, R. Fazio, A. Osterloh, and V. Vedral, Rev. Mod. Phys. 80, 517 (2008); F. Mintert, A. Carvalho, M. Kus, A. Buchleitner, Phys. Rep. 415, 207 (2005); W. K. Wootters, Phys. Rev. Lett. 80, 2245 (1998).

[60] S. Bose, Phys. Rev. Lett. 91, 207901 (2003).

[61] M. Menzel, Y. Mokrousov, R. Wieser, J. E. Bickel, E. Vedmedenko, S. Blugel, S. Heinze, K. vonBergmann, A. Kubetzka, and R. Wiesendanger, Phys. Rev. Lett. 108, 197204 (2012).
[62] P. Zanardi, H. T. Quan, X. Wang, and C. P. Sun, Phys. Rev. A 75, 032109 (2007); W. L. You, Y. W. Li, and S. J. Gu, Phys. Rev. E 76, 022101 (2007).

[63] A. J. Hearmon, F. Fabrizi, L. C. Chapon, R. D. Johnson, D. Prabhakaran, S. V. Streltsov, P. J. Brown, and P. G. Radaelli, Phys. Rev. Lett. 108, 237201 (2012).

[64] S. R. White, Phys. Rev. Lett. 69, 2863 (1992).

[65] U. Schollwöck, Rev. Mod. Phys. 77, 259 (2005).

[66] A. A. Nersesyan, A. O. Gogolin, and F. H. L. Essler, Phys. Rev. Lett. 81, 910 (1998).

[67] A. Luther and I. Peschel, Phys. Rev. B 12, 3908 (1975).

[68] I. Affleck and M. Oshikawa, Phys. Rev. B 60, 1038 (1999).

[69] N. M. Bogoliubov, A. G. Izergin, and V. E. Korepin, Nucl. Phys. B 275, 687 (1986).

[70] D. C. Cabra, A. Honecker, and P. Pujol, Eur. Phys. J. B 13, 55 (2000).

[71] P. Lecheminant, T. Jolicoeur, and P. Azaria, Phys. Rev. B 63, 174426 (2001).

[72] A. O. Gogolin, A. A. Nersesyan, and A. M. Tsvelik, Bosonization and Strongly Correlated Systems (Cambridge University Press, Cambridge, UK, 1998).

[73] Contrary to the Lanczos results in J. Vahedi and S. Mahdavifar, Eur. Phys. J. B 85, 171 (2012), we note that for sufficiently long chains chirality in ferromagnetic region $J_{1}<-44_{2}$ is nonzero for any $d \neq 0$, including for $B=0$. The discrepancy is due to the fact that in Lanczos simulations only small system sizes are accessible.

[74] M. Arlego, F. Heidrich-Meisner, A. Honecker, G. Rossini, and T. Vekua, Phys. Rev. B 84, 224409 (2011).

[75] A. K. Kolezhuk, F. Heidrich-Meisner, S. Greschner, and T. Vekua, Phys. Rev. B 85, 064420 (2012). 